\title{
Prévision des crues dans les petits bassins versants de montagne : prise en compte de la variabilité spatiale des pluies et des mécanismes de production par l'approche DPFT
}

\author{
J.Y. Rodriguez \\ EDF - DTG. Sce Ressources en Eau. B.P. 4348. 31029 Toulouse Cedex. FRANCE
}

Ch. Obled

ENSHMG-IMG. B.P. 53 X. 38041 Grenoble Cedex. FRANCE

D. Sempere-Torres

INSTITUT JAUME ALMERA. Ap. 30102. 08080 Barcelona. ESPAGNE

\section{Introduction}

\subsection{Le contexte : les besoins en prévision des crues}

Il est toujours important de connaître en temps réel l'état d'une rivière susceptible de menacer, par une montée rapide des eaux, des biens économiques ou des vies humaines. Il est encore plus intéressant d'anticiper cet état, de quelques heures à quelques jours quand cela est possible, pour prendre à temps des mesures adéquates.

Pour certaines grandes rivières ou systèmes fluviaux, on peut attendre que les volumes de crues soient déjà formés dans le réseau hydrographique, à l'amont, pour en déduire, en fonction de la propagation, l'évolution probable de la situation à l'aval, avec un délai suffisant... (cas de la Seine, de la Loire, du Rhône, de l'Isère).

Mais dans de nombreux cas, on se trouve en tête du réseau hydrographique, déjà très en amont, et on ne peut attendre que les débits se forment pour s'en protéger. Ceci est d'autant plus vrai que les bassins deviennent "petits " (de l'ordre de quelques dizaines ou centaines de $\mathrm{km}^{2}$ ), ce qui rend leur temps de réponse assez court. Souvent aussi, ces bassins supérieurs ont un relief plus marqué que le cours inférieur des grandes rivières, et cette topographie contribue encore à accélérer la propagation des écoulements. C'est le cas de nombreux petits bassins versants de montagne, et de beaucoup de fleuves côtiers le long de la frange méditerranéenne. Dans le cas de ces derniers, ils

Flow forecast in small mountainous watersheds :

Inclusion of rainfall and production mechanism spatial variabilities by the

FDTF-ERUHDIT approach

This paper condenses the last results obtained in rainfall-runoff modeling by the lumped and operational FTDF-ERUHDIT model. After placing the approach in relation to current needs, some tests and results obtained with synthetic data are presented (model adequacy if the transfer function is not supposed to be invariant, baseflow filtering capability, ...). The extension of this method to bi-inputs cases is also discussed. Finally, some researches on production functions, which may be from now fitted using FDTF-ERUHDIT results like an input-output problem, are summarized. Three production functions, belonging to the three great families currently used in hydrology, have been tested and compared. The introduction of index linked with rainfall fields or contributing areas seems to improve the results of this lumped and operational model. 

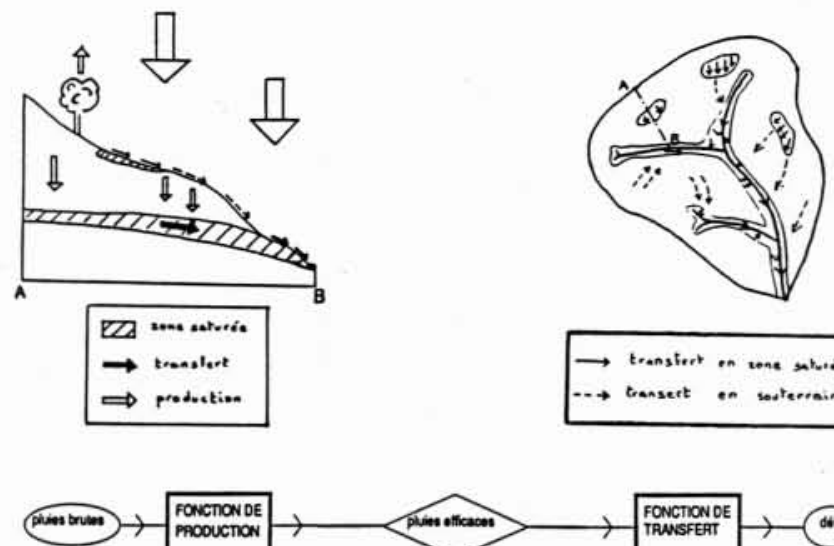

rovicnow ot
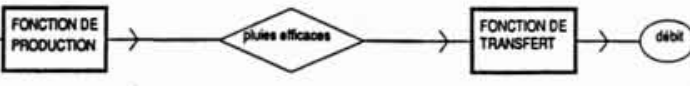

1. Analogie entre le système physique et le modèle de l'Hydrogramme Unitaire.

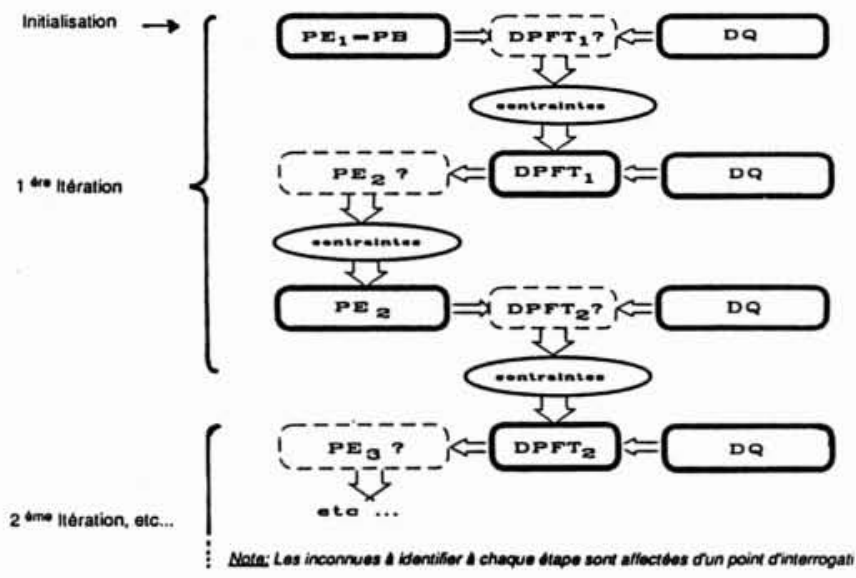

2. Schéma de fonctionnement de la DPFT. débouchent directement sur des plaines littorales étroites, et surtout fortement urbanisées (cas du Paillon à Nice, de l'Huveaune à Marseille, etc...). Dans d'autres cas, ce sont de petits affluents de systèmes fluviaux qui peuvent en cas de crues, en perturber la gestion (cas des affluents rive droite du Rhône) et obligent les services gestionnaires $(E D F, C N R, \ldots)$ à les prendre en compte. Enfin, dans les régions à fréquentation touristique l'occupation saisonnière mais intensive du lit majeur (camping-caravanning, canoë) par des populations extérieures à la région oblige à une grande vigilance. D'où la nécessité de systèmes opérationnels d'annonce de crues.

Ceux-ci s'articulent autour de trois sous-systèmes :

- un système de collecte des données (acquisition - transmission - archivage), qui donne une vue « instantanée » du bassin.

- Un système de prévision qui, à l'aide de modèles et d'informations météorologiques, propose une ou des projections dans l'avenir.

- Un système de dissémination des informations et des prévisions.

Un exemple de ces systèmes opérationnels est décrit par TOURASSE (1991).

C'est surtout au deuxième sous-système que nous nous sommes consacrés.

\subsection{Les pratiques et les modèles}

Pour des raisons évidentes, les services chargés de la prévision souhaitent disposer de modèles "simples", c'est-àdire assez économes en données, robustes, capables de fonctionner en mode dégradé si certaines données font défaut, et que l'on peut mettre en action sur alerte météorologique, sans nécessiter un fonctionnement continu en dehors des périodes d'alerte.

Cela conduit en général à l'utilisation de modèles globaux, ou systémiques, qui comportent 2 grandes familles :

— Les modèles de « stockage » où l'entrée pluie remplit un ou des réservoirs en séquence qui se vident selon des lois, linéaires ou non, pour fournir le débit à l'exutoire. - Les modèles à "fonction de transfert " qui décomposent le cycle hydrologique en deux phases. On distingue d'abord une phase de "production " des volumes ruisselés, dans laquelle sont regroupées toutes les « pertes » affectant la lame de " pluie brute » $\mathrm{PB}$ tombée sur le bassin. Ensuite, la lame restante, appelée "pluie efficace » $\mathrm{PE}$, est distribuée dans le temps et transférée à l'exutoire selon une "fonction de transfert" ou "hydrogramme unitaire", supposé indépendant du niveau de débit et invariant dans le temps. On essaye ainsi de représenter au premier ordre les processus hydrologiques du vrai système physique (figure 1). La structure de ce modèle est assez classique mais les pratiques pour l'identification de ses paramètres évoluent constamment (Dooge, 1973 ; Morel-SeYtouX, 1981 ; RodrigueZ, 1989).

L'approche proposée, appelée méthode DPFTERUHDIT est l'une de ces techniques d'identification. Dans la pratique courante, nombreuses sont les méthodes qui imposent a priori, de manière assez arbitraire et souvent sans remise en cause ultérieure, le calcul des pertes ou des pluies efficaces. Tout l'effort d'identification est alors limité à la fonction de transfert. La méthode DPFT considère, elle, que la fonction de transfert reste à identifier, mais que les pluies efficaces doivent l'être aussi et constituent une partie des inconnues du système (cf. figure 2). Une fois identifiées, elles serviront ensuite à calibrer une fonction de production qu'on pourra alors choisir parmi celles déjà proposées dans la littérature (une application est donnée dans SEMPERE-TORRES, 1990), au lieu de l'imposer a priori, comme cela se pratique d'habitude.

Cela mis à part, la méthode reprend diverses techniques déjà proposées antérieurement : utilisation d'un ensemble de données multi-événements (MAYs et CoLEs, 1980), ajustement « moyen " par moindres carrés avec régression en crête (Versiani, 1983),... Elle y ajoute une technique de travail sur les différences premières de débit - ou leurs variations - (GUILLOT et DUBAND, 1980), qui conduisent aux différences premières de la fonction de transfert (DPFT), beaucoup plus robuste statistiquement. 


\subsection{L'étude proposée}

Bien que déjà testée sur des données synthétiques (NALBANTIS, 1987: NALBANTIS et al., 1988), et utilisée de manière opérationnelle depuis plusieurs années par le Service Ressources en eau de la Division technique générale d'EDF (DUBAND et al., 1990), cette approche robuste méritait d'être développée dans 2 directions :

- tester la sensibilité des algorithmes aux hypothèses de la méthode (notamment l'invariance de la F.T.) et étendre leur application à des cas multi-entrées. Il s'agit alors de chercher à identifier les fonctions de transfert et les pluies efficaces de deux sous-bassins distincts, mais dont on ne connait qu'une sortie correspondant à leur exutoire commun (figure 3 ).

- Proposer, tester et comparer des modèles de production existant dans la littérature, et essayer de les améliorer et de mesurer la perte d'information propre à ce maillon faible de la modélisation hydrologique.

\section{Les résultats obtenus}

\subsection{Amélioration algorithmiques et tests d'adéquation de la DPFT}

La méthode a été largement testée sur des données générées artificiellement, c'est-à-dire dont on connaissait la structure de création et toutes les valeurs aux étapes intermédiaires. Quand ensuite on ne fournit à la méthode DPFTERUHDIT que les seules entrées et sorties, en demandant d'identifier les valeurs intermédiaires (pluie efficace, fonction de transfert), on peut alors comparer avec les vraies valeurs qui ont été utilisées lors de la génération, et qui servent de référence. Cette méthode permet de tester, d'une part, la capacité des différents algorithmes à retrouver les "vraies" valeurs intermédiaires, et de mesurer, d'autre part, leur sensibilité en présence de divers types d'erreurs (mesure, inadéquation du modèle,...).

On a d'abord recherché des algorithmes plus performants que les moindres carrés pour identifier aussi bien la fonction de transfert que les pluies efficaces. C'est ainsi que l'on s'est tourné vers la programmation linéaire, puis quadratique. Ces deux méthodes ont l'avantage d'inclure dans le calcul même des inconnues à identifier des contraintes de plausibilité comme :

- la fonction de transfert est toujours positive (un incrément de pluie ne peut induire qu'un accroissement de débit).

- Les pluies efficaces sont positives ou nulles (mais pas négatives !) et éventuellement toujours inférieures aux pluies brutes (si l'on est sûr de cette valeur d'entrée, c'està-dire, si l'on dispose d'un réseau de mesure suffisamment dense et fiable).

Auparavant, ces contraintes n'étaient appliquées qu'a postériori, de manière sous-optimale.
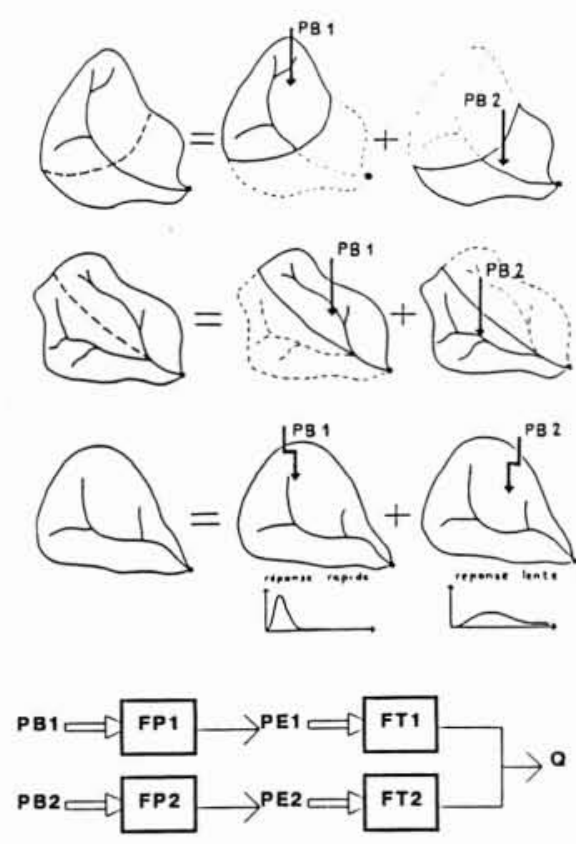

3. Exemple de problèmes justifiant une approche "Double Entrée " et schématisation proposée.

De même, on a testé la réponse du modèle quand, contrairement aux hypothèses utilisées, la fonction de transfert n'est pas invariante dans le temps mais présente une réponse plus raide, par exemple, aux fortes pluies, ou aux forts débits. On a pu vérifier que pour une F.T. de forme classique (loi gamma) ayant un pic au pas de temps 5 pour une durée totale de 15 , la translation du pic de plus ou moins un pas de temps, selon que les débits soient faibles ou forts, ne dégrade pas significativement les résultats d'identification. Par contre, cela devient sensible quand, pour un temps moyen au pic toujours égal à 5 , le vrai temps de montée passe de 3 à 7 selon que les débits soient forts ou faibles (cf. figure 4, page suiv.).

On démontre ainsi que, même dans des cas où il existerait une réponse faiblement non invariante (la "vraie" réponse du bassin pourrait varier d'un pas de temps autour de la réponse moyenne), la méthode DPFT permet, malgré l'hypothèse d'invariance sur la fonction de transfert, de reproduire et prévoir très convenablement les débits à l'exutoire.

Néanmoins, si certains auteurs ont constaté et étudié les variations des fonctions de propagation débit-débit dans les parties inférieures des cours d'eau (KEEFER et QUIVEY, 1974 ; BeCKer et KunDzewic, 1987), le problème de la détermination du degré d'invariance d'un bassin amont, de type pluie-débit, demeure.

On a aussi testé l'intérêt de filtrer le débit de base (la composante lente de l'hydrogramme), comme cela est fait couramment dans la pratique des modèles de type « hydro- 



(b) JeU JFT
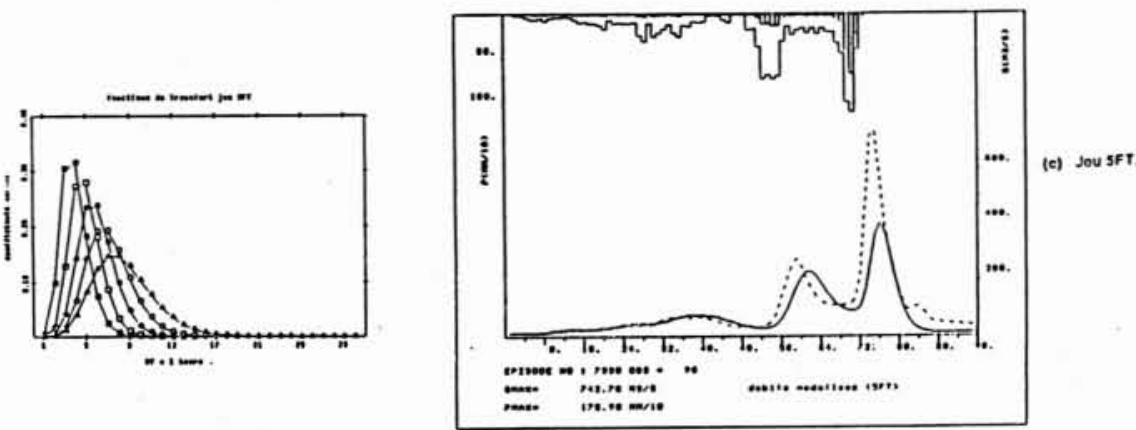

4. Test de sensibilité à l'hypothèse d' "Invariance de la Fonction de transfert 》 :

Exemple de crue :

générée avec 1 ou 3, ou 5 F.T. (d'autant plus raides que l'intensité de la pluie efficace est forte),

modélisée avec 1 seule F.T. invariante (on voit que les écarts croissent quand on s'écarte de l'hypothèse).

gramme unitaire ». Pour cela, une composante lente, non linéaire, a été rajoutée au débit de ruissellement. D'une part, nous avons traité avec l'approche DPFT les débits totaux, sans effectuer de filtrage préalable. D'autre part, nous avons préalablement filtré la composante lente, en utilisant, bien entendu, un modèle de filtrage différent de celui employé pour générer le débit de base, mais qui garde, néanmoins, des caractéristiques conceptuelles semblables. Bien que la méthode élimine déjà en partie cette composante, grâce à l'utilisation des différences premières, on a montré que dans certains cas, comme par exemple quand l'on soupçonne que le débit dit de "base " contribue de manière significative au volume de crue, un filtrage spécifique pouvait encore améliorer les performances.

\section{LA HOUILLE BLANCHE/N 5-1991}




\subsection{Extension de la DPFT à des cas bi-entrées}

Nous aurions pu considérer, lors de l'étude d'adéquation de la méthode DPFT à des cas où le bassin présente des F.T. non invariantes, qu'une des causes de cette noninvariance pourrait provenir d'une forte disparité des champs de pluie. Dans le cas des bassins de montagne, on sait qu'il y a un gradient pluviométrique directement lié au gradient daltitude. Mais ceci ne veut pas dire que tout épisode de crue suit ce champ moyen. On pourrait même affirmer, sans trop se tromper, qu'à l'intérieur d'un même épisode pluvieux, la morphologie du champ des précipitations peut varier considérablement d'un pas de temps à un autre.

Si nous raisonnons maintenant non plus en précipitation brute, mais en lame efficace, cette disparité peut éventuellement s'aggraver. Imaginons un bassin de montagne. Parmi les épisodes pluvieux de forte intensité moyenne, certains peuvent être, d'une part, plus ou moins efficaces selon l'altitude de la limite pluie-neige, son évolution dans le temps, la concentration de pluie sur la partie supérieure ou inférieure du bassin,... D'autre part, le transfert de la lame ruisselée, vue globalement, pourra être plus ou moins rapide selon la productibilité de chaque sous-bassin considéré.

Pour intégrer une partie de cette information spatiale, la méthode a été testée dans le contexte multi-entrées/monosortic. L'objectif était d'identifier les réponses distinctes de 2 sous-systèmes non jaugés, dont on ne connaissait que la sortie commune. Pour espérer identifier leurs fonctions de transfert propres, il faut d'abord que leurs entrées respectives soient raisonnablement différentes (partiellement décorrélées). On a pu montrer dans ce cas que l'identification des fonctions de transfert distinctes était possible à l'aide d'un algorithme itératif-alterné, grâce notamment au traitement multi-événements.

Cependant le second volet de la méthode, et le plus intéressant en pratique, est la déconvolution, on encore la reconstitution des séries de pluies efficaces. Ici, il y avait, pour chaque entrée, une série à déconvoluer. On a montré alors que l'on arrivait très rapidement à des systèmes indéterminés, comportant plus d'inconnues que d'équations. On ne peut résoudre ce problème qu'en introduisant des équations supplémentaires traduisant des hypothèses de comportement du bassin, ou des contraintes. Parmi les différents algorithmes mis au point et testés, celui qui exploite, à l'aide d'une fonction écart entre les 2 soussystèmes, le manque d'information fournit par la réponse moyenne (ce qui correspond à une forme d'orthogonalisation), s'est révélé le plus efficace (RodriguEZ, 1989).

Quelques simulations sur données générées ont montré que la nouvelle méthode bi-entrées ne pouvait apporter un plus par rapport à la version classique mono-entrée que si les processus de production étaient bien différents sur les deux sous-bassins. Cependant, les hypothèses de comportement imposées pour résoudre le problème inverse de déconvolution simultanée de deux séries de pluies efficaces introduisent un biais. Nous avons retrouvé ce résultat sur deux applications réelles. Lorsque le modèle global fournit des résultats "standard», cas du Gardon d'Anduze $\left(545 \mathrm{~km}^{2}\right)$ sur les Cévennes, la version bi-entrées, plus lourde, n'apporte aucune performance (RODRIGUEZ et al., 1989b). Par contre, sur la Petite Creuse à Puyrageaud $\left(840 \mathrm{~km}^{2}\right)$ sur l'Ouest du Massif central, la version monoentrée ne donnait pas de résultats satisfaisants (figure 5), mais le modèle semi-global a permis d'obtenir des résultats "standard" (Rodriguez et OBLED, 1990).

Néanmoins, une des conclusions de l'étude était le constat de l'incapacité des modèles de production «pluie brute - pluie efficace " connus à reproduire les séries de pluie efficace déconvoluées par l'approche DPFT. On s'est donc intéressé à ce deuxième volet.
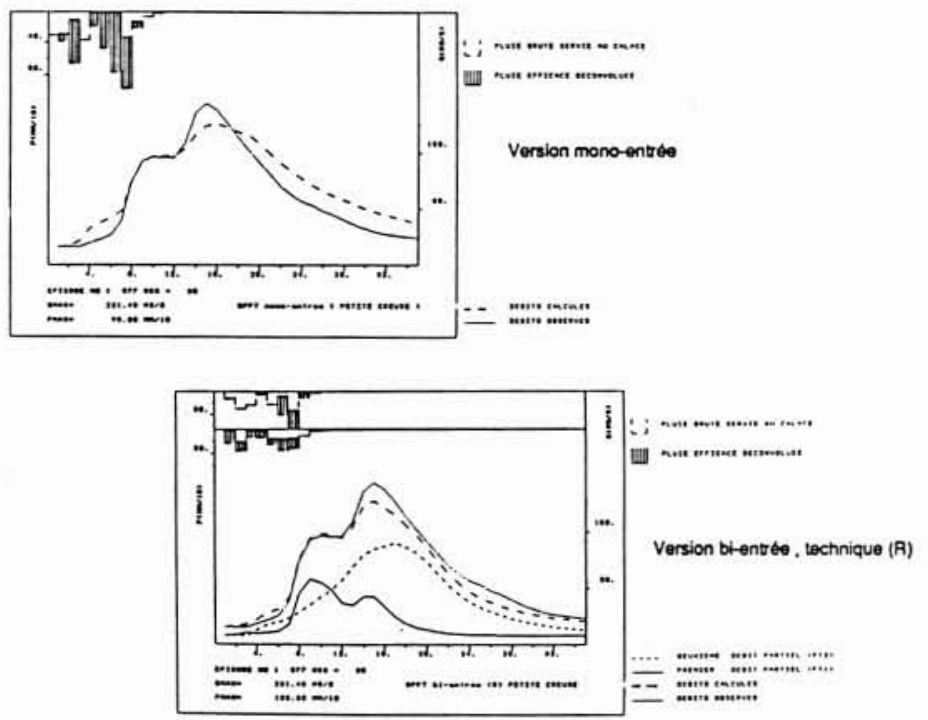

5. Exemple de crue reconstituée avec les versions mono et bi-entrée de la DPFT (Cas de la Petite Creuse à Puy-Rageaud $840 \mathrm{~km}^{2}$ ).

\subsection{Test et adaptation de modèles de production}

Arrivé à ce point, on a donc identifié des pluies efficaces par déconvolution, que nous appellerons PED, et qui reconstituent au mieux, combinées à la Fonction de Transfert F.T., elle aussi identifiée, les débits observés. Mais, pour que la chaîne soit complète, il faut être capable de relier ces pluies efficaces PED au pluies brutes $\mathrm{PB}$ par un modèle de production. Celui-ci, en usage opérationnel, donc sur une nouvelle crue, prendra en entrée les pluies brutes mesurées, calculera les pluies efficaces "modèle " résultantes PEM, qui serviront via la F.T. à prévoir les débits futurs.

Comme on l'a déjà précisé, le grand avantage de l'approche DPFT-ERUHDIT consiste à ne pas imposer a priori le choix de ce modèle de production. De plus, les pluies efficaces PED, fournissent non seulement un moyen 
Episode 7930
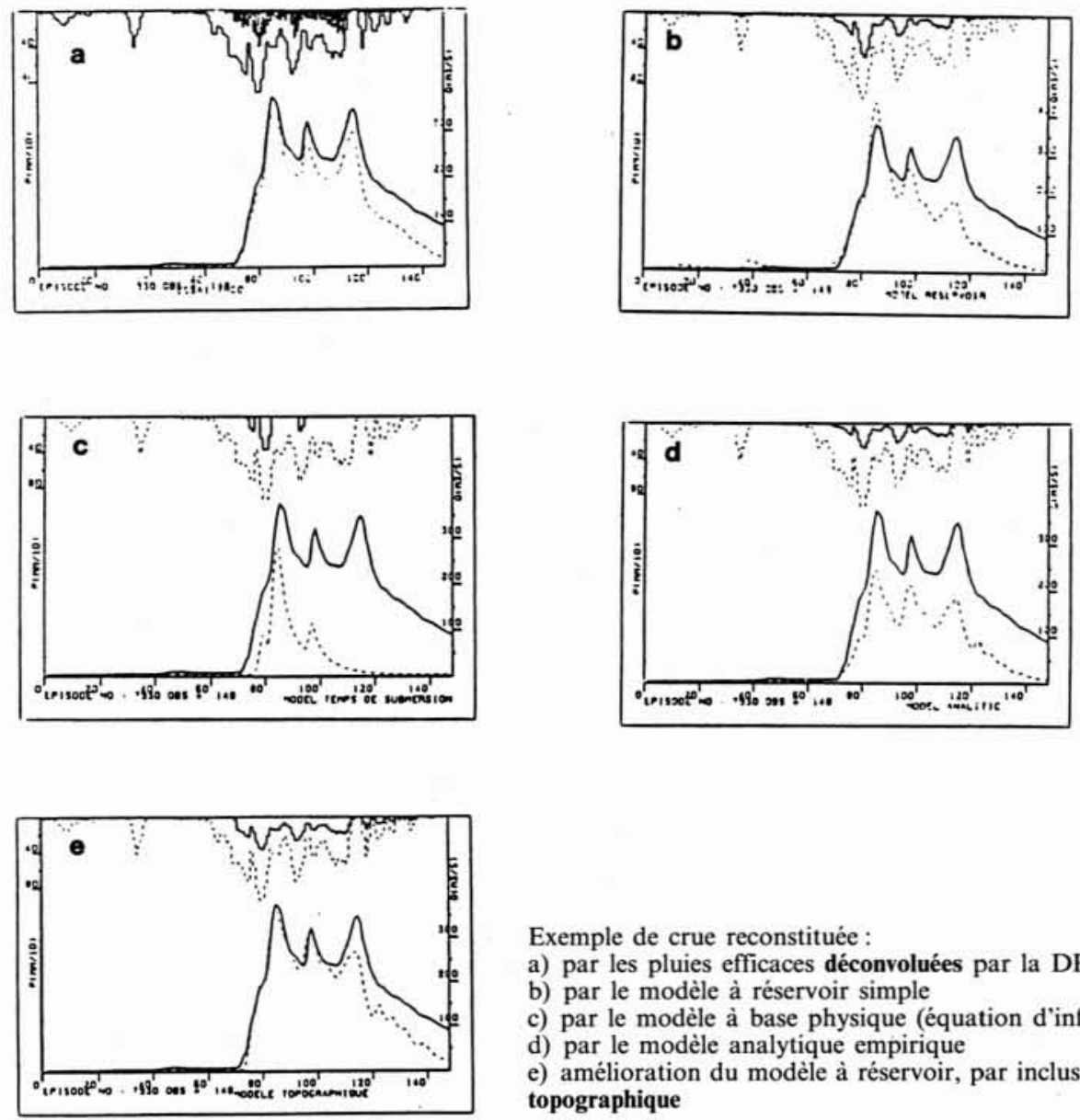

Exemple de crue reconstituée :

a) par les pluies efficaces déconvoluées par la DPFT (référence)

b) par le modèle à réservoir simple

c) par le modèle à base physique (équation d'infiltration)

d) par le modèle analytique empirique

e) amélioration du modèle à réservoir, par inclusion d'une composante topographique

6. Comparaison de Fonctions de Production.

pour ajuster et comparer différents modèles de production (RODRIGUEZ et al., 1989a), mais deviennent aussi une référence qui permet de mieux comprendre et mieux modéliser la structure et la dynamique de la fonction de production «idéale » (celle qui est capable de reproduire ces PED).

Dans une première étape, on s'est cantonné à la comparaison de modèles de production globaux. Nous supposons donc que le bassin répond globalement comme une seule entité à une $\mathrm{PB}$ supposée homogène spatialement). On a utilisé trois modèles, représentant trois grandes familles de ces "fonctions de perte".

- La première recherche une forme analytique optimale, de la forme

$$
P E=P B^{2} / P B+R
$$

où $R$ dépend des pluies et débits antérieurs. C'est une formulation classique et robuste (GUILLOT et Duband, 1980). Grâce à l'utilisation autorégressive des indices de pluies et débits antérieurs, et en dépit d'un nombre élevé de paramètres, elle ne nécessite pas d'initialisation. Ainsi, la simple utilisation du débit initial convient pour s'affranchir de ce problème, commun aux modèles fonctionnant par événements.

- La seconde est représentée par un modèle conceptuel où le bassin est assimilé à un réservoir qui se vidange et dont le déficit de remplissage pilote la production de pluie efficace (LORENT, 1975). Ce modèle présente l'inconvénient d'avoir besoin de " calculer " l'état de remplissage initial au début de l'épisode. Mais il possède un nombre réduit de paramètres, et reste donc facile à caler.

- Enfin, le dernier modèle utilisé est à base physique. Il étend les équations d'infiltration, déduites de la parcelle de terrain, à l'échelle du bassin versant (Morel-Seytoux, 1988).

Ceci suppose implicitement que le bassin puisse être décrit par le comportement d'une colonne de sol équivalente, où les paramètres caractérisant les propriétés du sol ont été remplacés par des "paramètres équivalents». Ce modèle a aussi besoin d'initialiser l'état de saturation du bassin. 
Ces trois modèles ont été comparés très soigneusement sur deux bassins: Le Gardon d'Anduze $\left(545 \mathrm{~km}^{2}\right)$ sur les Cévennes, et le Réal Collobrier $\left(70 \mathrm{~km}^{2}\right)$, sur le massif des Maures.

Une des conclusions principales est la nette inadéquation, à l'échelle du bassin, des modèles déduits des équations d'infiltration, pourtant présumées plus " physiques ». Nous avons vérifié que les équations ponctuelles ne sont pas valables à des échelles plus vastes, ceci étant certainement dû à la grande variabilité spatiale des propriétés des sols, qui ne peut pas être synthétisée par l'utilisation de "paramètres équivalents".

En revanche, les modèles à réservoir, même s'ils renoncent à être physiques, se montrent les mieux adaptés à la reproduction de la dynamique des pluies efficaces déconvoluées PED. Mais l'initialisation du modèle (état du réservoir au début de chaque crue) reste un problème qu'il faudra probablement résoudre par des techniques adaptatives.

Néanmoins, les résultats obtenus par les trois modèles de production sont équivalents, malgré des structures fort distinctes. Ils sont capables d'expliquer 50 à $60 \%$ de la variance des variations de débit, et 70 à $80 \%$ de celle des débits. De plus, les trois modèles présentent, à certains pas de temps, le même type de problèmes. Ceux-ci sont donc indépendants de la dynamique propre des fonctions de production proposées.

L'analyse de ces situations montre, qu'en général, il s'agit de pas de temps oủ la séquence temporelle de la lame moyenne de pluie reçue par le bassin (la pluie brute $P B$, unique information utilisée par le modèle, hormis le débit aux pas de temps précédents) n'informe pas suffisamment pour expliquer la dynamique de la pluie efficace (OBLED, 1989 ; Sempere-Torres, 1990). Ainsi, à certains pas de temps, les modèles de production globaux négligent de façon exagérée les variabilités spatiales des champs de pluie d'une part, et des propriétés de production du bassin d'autre part.

Ces constatations nous ont amené à explorer deux voies, visant à introduire une information morphologique complémentaire sous forme d'indices, afin de rester cohérents avec l'objectif opérationnel de l'approche DPFT.

Nous avons d'abord proposé un modèle qui combine l'information topographique du bassin - sous forme de l'indice $\operatorname{Ln}\{a / \operatorname{Tan} b\}$ de BEVEN et KIRKBY (1979) - et l'un des modèles globaux étudiés précédemment. On inclut ainsi explicitement le mécanisme de pluie efficace par excès de saturation, qui complète le mécanisme hortonnien d'efficacité par excès d'infiltration (SEMPERE-TORRES, 1990).

La deuxième voie explorée a consisté à introduire des indices de concentration de pluie (OBLED et RodRiguez, 1988), ce qui permet de caractériser la forme du champ pluvieux par rapport à la lame moyenne. Ces indices ont été utilisés comme facteur de correction des pluies efficaces calculées par l'un des modèles globaux (dans notre cas, le modèle à réservoir, qui avait fourni les meilleurs résultats). On obtient ainsi des pluies efficaces "nuancées " par le degré de concentration de la pluie, qui mesure en pratique le pourcentage du bassin concerné par le processus de production (SEMPERE-Torres et Obled, 1990).
Les résultats ainsi obtenus par l'utilisation de ces deux indices ont montré que si les améliorations introduites ne sont que significatives sur l'ensemble des épisodes, elles deviennent importantes quand on regarde la dynamique des reconstitutions, tout particulièrement celle des pics (figure 6).

Notons enfin qu'en phase de calibration des modèles de production, les résultats obtenus montrent qu'il est préférable d'utiliser des critères de similitude entre les pluies efficaces modélisées et déconvoluées, qu'entre les débits ou leur variations. Ceci corrobore l'intérêt de disposer d'une estimation de la pluie efficace, comme celle fournie par la méthode DPFT.

\section{Conclusion}

L'approche DPFT est une méthode itérative et alternée d'identification de la fonction de transfert et de déconvolution des pluies efficaces. L'étude menée à son sujet, dans le cadre du programme d'amélioration des prévisions des crues dans les bassins versants de montagne, s'est intéressée aux deux composantes de cette modélisation de type hydrogramme unitaire : le transfert et la production.

Pour le transfert, nous avons constaté, à l'aide de données générées, que la méthode pouvait s'adapter à des cas où la réalité s'éloignait des hypothèses d'invariance de l'hydrogramme unitaire. Par ailleurs, nous avons vérifié qu'elle filtrait correctement, grâce au traitement en différences premières, le débit de base. Mais si le volume de ce dernier représente une partie non négligeable du volume total de crue, un filtrage préalable pouvait encore améliorer les résultats.

La méthode a aussi été développée dans un contexte bi-entrées. On différencie ainsi les processus de production et de transfert de deux parties du bassin, en général, l'amont et l'aval. Nous avons montré que cette extension ne pouvait pas remplacer la méthode originale dans le cas où celle-ci fournissait des résultats "standard", mais pouvait permettre de les atteindre si la version monoentrée ne donnait pas les résultats escomptés.

Pour la partie production, nous avons pu calé trois modèles représentant trois grandes familles de fonctions perte: un modèle analytique, un modèle conceptuel à réservoir, et un modèle à base physique. Ceci est possible grâce à la disponibilité des pluies efficaces déconvoluées par la méthode DPFT.

Les trois modèles ont donné des résultats équivalents, plafonnant, en terme de variance expliquée des variations de débits, à $50-60 \%$. L'analyse de la dynamique des trois modèles nous a permis d'observer qu'aucun d'eux n'était capable de fournir une pluie efficace proche de celle obtenue par la DPFT quand la lame moyenne de pluie mesurée (ou brute) représentait mal le vrai champ de pluie. Ceci nous a conduit à introduire un indice de concentration de pluie. 
De plus, les trois modèles tendent à représenter des pluies efficaces produites par excès d'infiltration. Afin d'introduire le mécanisme de production par excès de saturation, nous avons combiné les modèles globaux avec une version simplifiée de l'indice topographique de BEVEN et KIRKBY (1979).

L'introduction de ces deux indices, faite séparément, nous a donné une amélioration des reconstitutions des crues, particulièrement marquée sur les pics. Les informations supplémentaires introduites se sont montrées fort prometteuses. Elles justifient que des recherches concernant leur approfondissement soient menées.

\section{Remerciements}

Cet article a présenté les différentes recherches effectuées à l'Institut de Mécanique de Grenoble sur l'opération "prévision des crues dans les petits bassins de montagne ", dans le cadre de l'action Risques naturels de la DATAR Rhône-Alpes (Programme 1986-1989). Nous remercions aussi D. DUBAND, d'Electricité de France - Division technique générale, co-responsable de l'étude, pour ses conseils et le suivi de cette recherche.

\section{Références}

BECKER A. et KUNDZEWICZ P.K. (1987). Nonlinear flood routing with multilinear models. Water Resources Research. Vol. 23, n 6. pp. 1043-1048.

BEVEN K.J. et KIRKBY M.J. (1979). A physically based variable contributing area model of basin hydrology. Hydrol. Sci. Bull. 24,1,3. pp. $42-69$.

Dooge J.C.I. (1973). Linear Theory of hydrological systems. U.S. Dep. Agric. Whashington D.C. Tech. Bull. $\mathrm{n}^{\circ} 1468.327$ pages.

Duband D.,N albantis I., Obled Ch., Rodriguez J.Y. et TOURASSE P. (1990). Unit Hydrograph revisited trough differencing and deconvolution on multi-event data sets : The FDTF approach. Publ IAHS. $\mathrm{n}^{\circ} 190$. pp. 377-390.

Guillot P. et Duband D. (1980). Fonctions de transfert pluiedébit sur des bassins versants de l'ordre de $1000 \mathrm{~km}^{2}$. Publ IAHS. nं 129. pp. 177-186.

KeEFER T.N. et QUIVEY R.S. (1974). Multiple linearization flow routing model. J. Hydraul. Div. ASCE. Vol. 100. Hy 7. pp. 1031-1046.

LORENT B. (1975). Tests of different rives flow predictions, in G.C. Vansteemkiste (Ed.). Modeling and Simulation of Water
Resources Systems. Nort-Holland Publishing Company. Amsterdam. Pays-Bas. pp. 233-241.

MaYs L.W. et Coles L. (1980). Optimization on Unit Hydrograph Determination. J. Hydraul. Div. ASCE. Vol. 106. Hy 1. pp. 85-97.

MoRel-Seytoux H.J. (1981). Optimization methods in RainfallRunoff modeling. in Rainfall-Runoff Relationship. pp. 487506. Proc. of the Intern. Symp. on Rainfall-Runoff Modeling. May, 18-21, 1981. Mississipi State University. EtatsUnis d'Amérique.

MOREL-SEYTOUX H.J. (1988). Recipe for simple but physically based modeling of the infiltration and local runoff processes. in H.J. Morel-Seytoux et al. (Eds). Proc. 8th Annual Hydrological Days. Hydrological Days Publications. Fort Collins. Colorado. Etats-Unis d'Amérique. pp. 226-247.

NALBANTIS I. (1987). Identification de modèles pluie-débit du type Hydrogramme Unitaire: Développements de la méthode DPFT et validation sur données simulées avec et sans erreurs. Thèse INPG. Grenoble. France.

Nalbantis I., Obled Ch. et Rodriguez J.Y. (1988). Modélisation pluie-débit : validation par simulation de la méthode DPFT. La Houille Blanche. 5/6. pp. 415-424.

OBLED Ch. (1989). Reflexions on rainfall information requirements for operational rainfall-runoff modelling. Int. Symp. on Hydrol. Application of Weather Radar. Univ. of Salford. August. 14-17. paper J4.

OBLed Ch. et Rodriguez J.Y. (1988). La distribution spatiale des précipitations et son rôle dans la transformation pluiedébit. La Houille Blanche. 5/6. pp. 467-474.

Rodriguez J.Y. (1989). Modélisation pluie-débit par la méthode DPFT : développements de la méthode initiale et extension à des cas bi-entrées. Thèse INPG. Grenoble. France.

Rodriguez J.Y., Sempere-Torres D. et Obled Ch. (1989a). Nouvelles perspectives de développement dans la modélisation des pluies efficaces par application de la méthode DPFT. IAHS Publ. no. 181. pp. 235-243.

Rodriguez J.Y., Sempere-Torres D. et Obled Ch. (1989b). Extension of Lumped Operational rainfall-runoff models to semi-lumped modelling: the case of the DPFTERUHDIT approach. Int. Symp. on Hydrol. Application of Weather Radar. Univ. of Salford. August. 14-17. Paper M2.

Rodriguez J.Y. et Obled Ch. (1990). Prise en compte de la variabilité spatiale des pluies efficaces par un modèle semiglobal: extension de la méthode DPFT à des cas bientrées. Publ. IAHS. n 193. pp. 635-644.

Sempere-Torres D. (1990). Calcul de la lame d'eau ruisselée dans la modélisation pluie-débit: limitations des approches globales et introduction simplifiée de la topographie et de la variabilité spatiale des pluies. Applications aux bassins versants du Gardon d'Anduze et du Réal Collobrier. Thèse INPG. Grenoble. France.

Sempere-Torres D. et Obled Ch. (1990). Modélisation pluiedébit en bassins à relief accidenté : Le rôle de la variabilité spatiale des pluies. Publ. IAHS. n' 193. pp. 645-654.

Tourasse P. (1991). Prévision et télésurveillance hydrométéorologique à EDF. La Houille Blanche. $\mathrm{n}^{\circ} 2 / 1991$. pp. 149154.

VeRSIANI B. (1983). Modélisation de la relation pluie-débit pour la prévision de crues. PhD. INPG. Grenoble. France. 